\title{
POLYNOMIALS WITH NONNEGATIVE COEFFICIENTS
}

\author{
R. W. BARNARD, W. DAYAWANSA, K. PEARCE, AND D. WEINBERG
}

(Communicated by Andrew Odlyzko)

\begin{abstract}
The authors verify the conjecture that a conjugate pair of zeros can be factored from a polynomial with nonnegative coefficients so that the resulting polynomial still has nonnegative coefficients. The conjecture was originally posed by A. Rigler, S. Trimble, and R. Varga arising out of their work on the Beauzamy-Enflo generalization of Jensen's inequality. The conjecture was also made independently by B. Conroy in connection with his work in number theory. A crucial and interesting lemma is proved which describes general coefficient-root relations for polynomials with nonnegative coefficients and for polynomials for which the case of equality holds in Descarte's Rule of Signs.
\end{abstract}

\section{INTRODUCTION}

Can a conjugate pair of zeros be factored from a polynomial with nonnegative coefficients so that the resulting polynomial still has nonnegative coefficients?

This question has attracted considerable attention during the last few years because of its seemingly elementary nature and its potential for applications in number theory and control theory. A proposed answer to this question arose as a conjecture by Rigler, Trimble, and Varga out of their work in [6], where explicit bounds were determined for the constants occurring in the BeauzamyEnflo generalization [1,2] of Jensen's Inequality. An affirmative answer to this question was conjectured independently by B. Conrey in connection with some of his work in number theory. Conrey announced the conjecture at the annual West Coast Number Theory Conference held in December 1987.

The main theorem in this note gives a positive answer to the question. This is proved in $\S 2$. The principal ingredients of the proof are an idea from index theory, classical properties of polynomials, and two lemmas that describe general coefficient-root relationships for polynomials with nonnegative coefficients and for polynomials for which the case of equality holds in Descartes' Rule of Signs (see [5]). We also prove a corollary of the main theorem, which describes the region into which certain zeros can be moved while preserving the nonnegativity of the coefficients.

Received by the editors September 21, 1989 and, in revised form, September 11, 1990.

1980 Mathematics Subject Classification (1985 Revision). Primary 30C10, 30C15. 
Consider the polynomial defined by $P_{N}(z)=1+z^{N}$. We note that

$$
P_{N}(z)=\prod_{l=0}^{N-1}\left(1-\frac{z}{e^{i\left(\frac{\pi}{N}+\frac{2 \pi l}{N}\right)}}\right)=\left(1-2 \cos \theta_{0} z+z^{2}\right) \sum_{k=0}^{N-2} b_{k} z^{k}
$$

has $b_{k}>0,0 \leq k \leq N-2$, if $\theta_{0}=\pi / N$, while any other choice for $\theta_{0}$, $\pi / N<\theta_{0} \leq \pi$, produces a factor with some negative $b_{k}$ coefficients.

Thus, one initial suggestion for the general question of factoring out a conjugate pair of zeros was to factor out a pair of zeros with greatest real part. The authors have used fairly straightforward arguments to show that if the degree of the polynomial is less than or equal to 5 , then a conjugate pair of zeros of greatest real part can be factored out, and the resulting polynomial will still have nonnegative coefficients. However, if

$$
p(z)=140+20 z+z^{2}+1000 z^{3}+950 z^{4}+5 z^{5}+20 z^{6}
$$

with $z_{0}, \bar{z}_{0}$ approximately equal to $0.392+6.390 i, 0.392-6.392 i$, resp., as the conjugate pair of zeros of greatest real part, then the resulting factors are $\left(z-z_{0}\right)\left(z-\bar{z}_{0}\right)$ and $\left(140.1+22.3 z-1.53 z^{2}+1000 z^{3}+966.7 z^{4}\right)$.

The other choice for which zeros to factor out, suggested by the example $P_{N}$ in (1), is the pair determined by the zero in the upper halfplane with smallest positive argument. Indeed, in this paper we prove the following:

Theorem 1.1. Let $p$ be a polynomial of degree $N, p(0)=1$, with nonnegative coefficients and zeros $z_{1}, z_{2}, \ldots, z_{N}$. For $t \geq 0$ write

$$
p_{t}(z)=\prod_{\substack{1 \leq j \leq N \\\left|\operatorname{Arg} z_{j}\right|>t}}\left(1-z / z_{j}\right) .
$$

Then if $p_{t} \neq p$, all of the coefficients of $p_{t}$ are positive.

Remark 1 . We note that a slightly sharper version of Theorem 1.1 is true. Suppose that the given polynomial $p$ has nonnegative coefficients and that we divide it by a single quadratic factor corresponding to any conjugate pair of zeros of smallest argument in magnitude. Then the quotient polynomial has nonnegative coefficients. Furthermore, if the conjugate pair of zeros is the unique pair of zeros of smallest argument in magnitude or if $p$ has strictly positive coefficients, then the quotient polynomial has strictly positive coefficients. The example $p(z)=\left(1+z^{2}\right)^{2}$ shows the necessity of these conditions. The sharpened version of the theorem under the uniqueness hypothesis follows from the proof of the main theorem. Now let us consider the case when several complex conjugate pairs of zeros of $p$, say $\left\{z_{i}, \bar{z}_{i}\right\}_{i=1, \ldots, k}$, have the smallest argument in magnitude and assume that all coefficients of $p$ are strictly positive. A local perturbation can be made of $z_{1}$ and $\bar{z}_{1}$ to $w_{1}$ and $\bar{w}_{1}$ such that the argument of $w_{1}$ is less than that of $z_{1}$. Under the local perturbation, the polynomial $p$ changes to $q$, which also has strictly positive coefficients. The sharpened version of the theorem under the uniqueness hypothesis implies that $q /\left(z-w_{1}\right)\left(z-\bar{w}_{1}\right)$ 
has strictly positive coefficients. Since $p /\left(z-z_{1}\right)\left(z-\bar{z}_{1}\right)=q /\left(z-w_{1}\right)\left(z-\bar{w}_{1}\right)$, the desired conclusion follows. Let $p$ be the polynomial referenced in the statement of Theorem 1.1. Let $t \geq 0$ and let $\left\{z_{1}, \ldots, z_{j_{t}}\right\}$ be the set of zeros of $p$ in the upper halfplane for which $0 \leq \operatorname{Arg}\left(z_{k}\right) \leq t, 0 \leq k \leq j_{t}$, indexed by order of increasing argument. The formal statement of Theorem 1.1 follows by successively removing from $p$ each pair of conjugate zeros $\left\{z_{k}, \bar{z}_{k}\right\}$, $k=1,2,3, \ldots, j_{t}$ and observing that removing the last such pair of conjugate zeros necessarily produces a factored quotient that has strictly positive coefficients.

Corollary 1.1. Let $p$ be a real polynomial with nonnegative coefficients, and let $z_{0}$ be a zero of $p$ in the upper halfplane with smallest positive argument. Then,

$$
p(z)=\sum_{k=0}^{N} a_{k}\left(z_{0}\right) z^{k}=\left(z-z_{0}\right)\left(z-\bar{z}_{0}\right) \sum_{k=0}^{N-2} b_{k} z^{k},
$$

where $a_{k}\left(z_{0}\right) \geq 0$. If $z_{1}$ is any number such that

$$
\left|z_{1}\right| \geq\left|z_{0}\right| \text { and } \operatorname{Re}\left\{z_{1}\right\} \leq \operatorname{Re}\left\{z_{0}\right\}
$$

then

$$
\sum_{k=0}^{N} a_{k}\left(z_{1}\right) z^{k}=\left(z-z_{1}\right)\left(z-\bar{z}_{1}\right) \sum_{k=0}^{N-2} b_{k} z^{k}
$$

has $a_{k}\left(z_{1}\right) \geq a_{k}\left(z_{0}\right)$.

Proof. The proof follows directly by noting, for $z_{0}=r e^{i \theta}=x+i y$,

$$
\begin{aligned}
\sum_{k=0}^{N} a_{k}\left(z_{0}\right) z^{k} & =\left(r^{2}-2 r z \cos \theta+z^{2}\right) \sum_{k=0}^{N-2} b_{k} z^{k} \\
& =\left(x^{2}+y^{2}-2 x z+z^{2}\right) \sum_{k=0}^{N-2} b_{k} z^{k}
\end{aligned}
$$

and comparing coefficients.

It has been recently noted by R. Evans and P. Montgomery [4] that in the special case when $p$ is the polynomial $P_{N}$ defined in (1) that the corresponding reduced $p_{t}$ polynomials are all strictly unimodal (in their coefficients). Also, R. Evans and J. Greene [3] have shown for polynomials $p$ of the form $p(z)=$ $\left(z^{M k}-1\right) /\left(z^{k}-1\right)$ that the corresponding reduced $p_{t}$ polynomials all have positive coefficients.

\section{Lemmas and Proof of Theorem 1.1}

Notation. Let $f$ denote a nonconstant polynomial with real coefficients. $\alpha(f)$ denotes the number of positive zeros of $f$, counted according to multiplicity, $\beta(f)$ denotes the number of positive critical points of $f$, counted according to multiplicity, and $\gamma(f)$ denotes the number of sign changes among the coefficients of $f$. Let $\delta(f)=\gamma(f)-\gamma\left(f^{\prime}\right)$ and note $\delta(f)=0$ or 1 . 
Before we give the proof of Theorem 1.1 we separate from it two lemmas that give a general insight into polynomial coefficient-root structure.

Lemma 2.1. Let $p$ be a real polynomial with nonnegative coefficients, and let $z_{0}=e^{i \theta_{0}}$ be a zero of $p$ in the upper halfplane with smallest positive argument. Suppose that $z_{0}$ is the unique zero of $p$ on the ray $\left\{r e^{i \theta_{0}}: 0<r<\infty\right\}$. Then for each nonnegative integer $k, \operatorname{Im}\left(p\left(t e^{i \theta_{0}}\right) / e^{i(k+1) \theta_{0}}\right)$ is a polynomial in $t$ for which the number of positive roots, counted according to multiplicity, is equal to the number of sign changes among its coefficients.

Lemma 2.2. Let $g$ and $h$ be two real polynomials with $h(z)=a_{n} z^{n}+\cdots+a_{N} z^{N}$ where $a_{n}>0, n<N$, and the degree of $g$ is less than $n$ with the leading coefficient of $g$ being negative. If $f$ is the polynomial defined by $f=g+h$ and the number of positive roots of $f$, counted according to multiplicity, is equal to the number of sign changes among the coefficients of $f$, then $h\left(z_{j}\right)$ is positive at each of the positive zeros, $z_{j}$, of $f$.

We note to prove Theorem 1.1 it suffices, by a successive reduction argument, to show that if we remove from $p$ a conjugate pair of zeros of smallest argument in the absolute value, then the resulting polynomial still has nonnegative coefficients.

Proof of Theorem 1.1. Let $p$ be a real polynomial with nonnegative coefficients, and let $z_{0}=r_{0} e^{i \theta_{0}}$ be a zero of $p$ in the upper halfplane with smallest positive argument. Then

$$
p(z)=\sum_{k=0}^{N} a_{k} z^{k}=\left(1-\frac{2 \cos \theta_{0} z}{r_{0}}+\frac{z^{2}}{r_{0}^{2}}\right) \sum_{k=0}^{N-2} b_{k} z^{k}
$$

with $a_{k} \geq 0,0 \leq k \leq N$. We may assume $a_{N}>0$. Let $\left\{r_{l} e^{i \theta_{l}}\right\}$ be the zero set of $p$ with $0<\theta_{0} \leq\left|\theta_{l}\right|$ for each $l$. We may assume $r_{0}=1$ in (2) by using $p\left(r_{0} z\right)$. Since the coefficients of $p$ depend continuously on the zeros of $p$, we may assume that $p$ has only one zero, say $z_{0}=e^{i \theta_{0}}$, on the ray $\left\{r e^{i \theta_{0}}: 0<r<\infty\right\}$ and that this zero is simple. Otherwise, by a variation of the coefficients followed by a local perturbation of the zeros, a sequence of polynomials with strictly positive coefficients and with this property can be chosen so that they converge to $p$. We prove that $b_{k}>0$ for $0 \leq k \leq N-2$.

Now

$$
\begin{aligned}
\sum_{k=0}^{N-2} b_{k} z^{k} & =\frac{p(z)}{1-2 \cos \theta_{0} z+z^{2}} \\
& =\sum_{k=0}^{\infty} \frac{\sin (k+1) \theta_{0}}{\sin \theta_{0}} z^{k} \sum_{k=0}^{N} a_{k} z^{k}=\sum_{k=0}^{N-2}\left(\sum_{l=0}^{k} \frac{\sin (l+1) \theta_{0}}{\sin \theta_{0}} a_{k-l}\right) z^{k}
\end{aligned}
$$


This gives

$$
b_{k}=\sum_{l=0}^{k} \frac{\sin (l+1) \theta_{0}}{\sin \theta_{0}} a_{k-l} .
$$

Noting that

$$
z^{N} p(1 / z)=\sum_{k=0}^{N} a_{N-k} z^{k}=\left(z^{2}-2 \cos \theta_{0} z+1\right) \sum_{k=0}^{N-2} b_{N-2-k} z^{k},
$$

it suffices to show that $b_{k}>0$ for $0 \leq k \leq[(N-2) / 2]$. From (3) if $\sin (l+1) \theta_{0}>0$ for $0 \leq l \leq k$, i.e., when $0<\theta_{0}<\pi /(k+1)$ it follows that $b_{k}>0$. Also, if all the zeros of $p$ are in the closure of the left halfplane, then the result is clear because $p$ can be put into the form:

$$
p(z)=\prod\left(1-\frac{2 \cos \theta_{l}}{r_{l}} z+\frac{z^{2}}{r_{l}^{2}}\right) \prod\left(1+\frac{z}{r_{m}}\right) .
$$

Thus, we can assume

$$
\pi /\left(\left[\frac{N-2}{2}\right]+1\right)=\pi /\left(\left[\frac{N}{2}\right]\right) \leq \theta_{0}<\frac{\pi}{2} .
$$

We assume that there is a $k$ with

$$
b_{k} \leq 0
$$

to reach a contradiction.

Let $f_{0}$ be defined by

$$
\begin{aligned}
f_{0}(t) & =\operatorname{Im}\left(p\left(t e^{i \theta_{0}}\right) / e^{i(k+1) \theta_{0}}\right) \\
& =-\sum_{l=0}^{k} a_{l}\left[\sin (k+1-l) \theta_{0}\right] t^{l}+\sum_{l=k+2}^{N} a_{l}\left[\sin (l-k-1) \theta_{0}\right] t^{l} .
\end{aligned}
$$

Lemma 2.1 implies that $\alpha\left(f_{0}\right)=\gamma\left(f_{0}\right)$; furthermore, the proof of Lemma 2.1 implies that $\alpha\left(f_{0}\right)=\gamma\left(f_{0}\right)=m$ or $m+1$, where $m$ is the unique integer for which $m \pi / N<\theta_{0} \leq(m+1) \pi / N$.

We observe that the function $f_{0}$ defined in (6) satisfies the hypothesis of Lemma 2.2 with $f_{0}=g+h$ where $g(t)=-\sum_{l=0}^{k} a_{l}\left[\sin (k+1-l) \theta_{0}\right] t^{l}$ and $h(t)=\sum_{l=k+2}^{N} a_{l}\left[\sin (l-k-1) \theta_{0}\right] t^{l}$. Since $\alpha\left(f_{0}\right)=\gamma\left(f_{0}\right)=m$ or $m+1$, it follows from a partitioning argument, that the leading coefficient of $g(t)$ is negative and that the coefficient of the term of smallest degree of $h(t)$ is positive. For otherwise, we have $\gamma\left(f_{0}\right) \leq m-1$, whereas the proof of Lemma 2.1 implies that $\alpha\left(f_{0}\right) \geq m$. Now since $f_{0}(1)=0$, Lemma 2.2 gives that $h(1)>0$, which implies that $g(1)<0$. But using (3), it follows that $g(1)=-b_{k} \sin \theta_{0}$, which contradicts the assumption in (5). Our main theorem follows. 


\section{Proofs of lemmas}

Proof of Lemma 2.1. Let

$$
p(z)=\sum_{k=0}^{N} a_{k} z^{k}
$$

with $a_{k} \geq 0, a_{N}>0$. Consider the function defined by

$$
F(z)=z^{-k-1} p(z)=\sum_{l=0}^{N} a_{l} z^{l-k-1}
$$

having the same zeros as $p$. Let $f_{0}$ be defined by

$$
\begin{aligned}
f_{0}(t) & =\operatorname{Im}\left[t^{k+1} F\left(t e^{i \theta_{0}}\right)\right]=\operatorname{Im}\left(p\left(t e^{i \theta_{0}}\right) / e^{i(k+1) \theta_{0}}\right) \\
& =-\sum_{l=0}^{k} a_{l}\left[\sin (k+1-l) \theta_{0}\right] t^{l}+\sum_{l=k+2}^{N} a_{l}\left[\sin (l-k-1) \theta_{0}\right] t^{l} .
\end{aligned}
$$

We observe from the hypothesis that $F$ has no zeros between the rays defined by $\left\{t e^{i \theta_{0}}: 0<t<\infty\right\}$ and $\left\{t e^{-i \theta_{0}}: 0<t<\infty\right\}$. Motivated by this, let $\Gamma=$ $\Gamma^{+} \cup \bar{\Gamma}^{+}$be the curve shown in Figure 1, which is symmetric about the reals with $\Gamma^{+}=\bigcup_{l=1}^{5} \Gamma_{l}$ where $\Gamma_{1}=\left\{R e^{i \theta}: 0 \leq \theta \leq \theta_{0}\right\}, \Gamma_{2}=\left\{t e^{i \theta_{0}}: 1+s \leq t \leq R\right\}$, $\Gamma_{3}=\left\{z_{0}+s e^{i \theta}: \theta_{0}-\pi \leq \theta \leq \theta_{0}\right\}, \Gamma_{4}=\left\{t e^{i \theta_{0}}: r \leq t \leq 1-s\right\}$, and $\Gamma_{5}=\left\{r e^{i \theta}: 0 \leq \theta \leq \theta_{0}\right\}$ and $r, s$, and $1 / R$ are chosen sufficiently small.

By Descartes' Rule of Signs, $\alpha\left(f_{0}\right) \leq \gamma\left(f_{0}\right)$. We show that if $\alpha\left(f_{0}\right)<\gamma\left(f_{0}\right)$, then the index of $F$ with respect to $\Gamma$ about any interior point is positive, implying the existence of a zero of $F$ inside $\Gamma$. This contradicts that $\theta_{0}$ is the smallest positive argument of the zeros of $p$ in the upper halfplane.

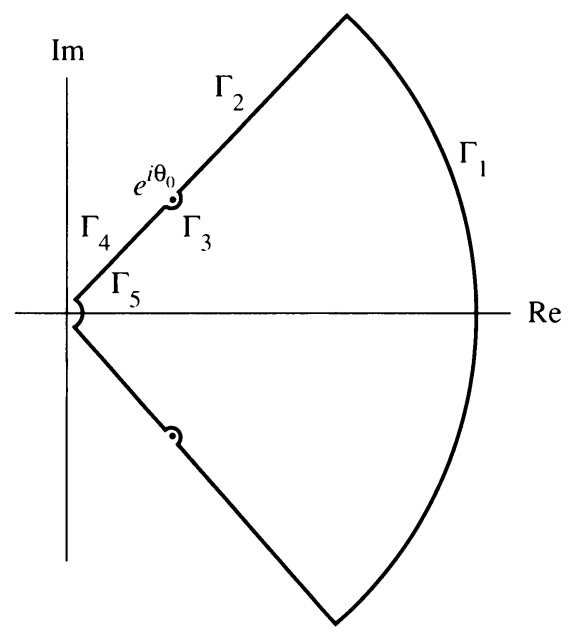

FIGURE 1 
The function $F$ has the form

$$
F(z)=a_{0} z^{-k-1}+\cdots+a_{k+1}+a_{k+2} z+\cdots+a_{N} z^{N-k-1} .
$$

From symmetry we can examine $\Delta \arg F(z)$, the change in the argument of $F$, as $z$ traverses $\Gamma^{+}$. Let $\Delta_{l}$ equal $\Delta \arg F(z)$ as $z$ traverses $\Gamma_{l}$. For $R$ sufficiently large, the term $a_{N} z^{N-k-1}$ in (7) dominates so that we have $\Delta_{1}=$ $(N-k-1) \theta_{0}+O(1 / R)$. For $r$ sufficiently small and positive, the term $a_{0} z^{-k-1}$ in (7) dominates so that $\Delta_{5}=-(-k-1) \theta_{0}+O(r)=(k+1) \theta_{0}+O(r)$, noting the clockwise transversal of $\Gamma_{5}$. Hence,

$$
\Delta_{1}+\Delta_{5}=N \theta_{0}+O(1 / R)+O(r)
$$

for $1 / R$ and $r$ sufficiently small. Let $\Gamma_{6}=\left\{t e^{i \theta_{0}}: r \leq t \leq R\right\}$, noting that $\Gamma_{2} \cup \Gamma_{3} \cup \Gamma_{4}$ approaches $\Gamma_{6}$ as $s \rightarrow 0$. For notational convenience, let $\widehat{\Delta}_{6}$ denote $\lim \left(\Delta_{2}+\Delta_{3}+\Delta_{4}\right)$ as $s \rightarrow 0$. We observe that a change in argument of $-\pi$ accounts for the change in argument determined by traversing $\Gamma_{3}$ for $s$ sufficiently small, i.e., $\Delta_{3}=-\pi+O(s)$. We make this observation to assure that $\Delta_{3}$ is counted only once in determining an upper bound for $\Delta_{6}$.

A bound for $\widehat{\Delta}_{6}$ can be determined by the maximum number of times the image of $\Gamma_{6}$ crosses the real axis. Descartes' Rule of Signs says that $\alpha\left(f_{0}\right)$ is bounded above by $\gamma\left(f_{0}\right)$. Since $a_{l} \geq 0$ for each $l, \gamma\left(f_{0}\right)$ is determined by the number of sign changes in $\sin l \theta_{0}$ as $l$ goes from $-k-1$ to $N-k-1$, i.e., over a range of length $N$. It is clear that there exists an integer $m$ such that

$$
\frac{m \pi}{N}<\theta_{0} \leq \frac{(m+1) \pi}{N} .
$$

Since the number of sign changes as $l \theta_{0}$ varies over an $N \theta_{0}$ range is determined by $N \theta_{0} / \pi$, it follows that $\gamma\left(f_{0}\right) \leq m+1$. In counting $\alpha\left(f_{0}\right)$ and $\gamma\left(f_{0}\right)$ if $a_{0} \sin (k+1) \theta_{0}=0$, then we factor out the leading power of $t^{l}$ in $f_{0}$. So, we are only considering positive zeros of $f_{0}$.

If $\alpha\left(f_{0}\right)<\gamma\left(f_{0}\right) \leq m+1$, then since Descartes' Rule of Signs implies that $\gamma\left(f_{0}\right)-\alpha\left(f_{0}\right)$ is even, we must have $\alpha\left(f_{0}\right)$ strictly less than $m$, say $m_{1}$ with $0 \leq m_{1}<m$. It follows that $\widehat{\Delta}_{6}<m_{1} \pi+\pi \leq m \pi$. Thus by using (7), (8), and (9), we obtain

$$
\Delta \arg F(z) \geq 2 \sum_{l=1}^{5} \Delta_{l}>2\left(N \theta_{0}-\left(m_{1}+1\right) \pi\right) \geq 0,
$$

for $1 / R, r$, and $s$ sufficiently small. This would imply that $\Gamma$ encloses a zero of $F$, contradicting the construction of $\Gamma$. This proves Lemma 2.1 .

To prove Lemma 2.2 we need the following auxiliary lemmas. In the proofs of the lemmas, we let $x_{1}(f) \leq x_{2}(f) \leq \cdots$ denote the positive zeros of a function $f$, indexed according to multiplicity, and set $x_{0}(f)=0$.

Lemma A. We have $\alpha(f) \leq \beta(f)+\delta(f) \leq \gamma(f)$, so the positive zeros and positive critical points of $f$ interlace if $\alpha(f)=\gamma(f)$. 
Proof. If $\alpha(f)=0$, then the first inequality holds trivially. Suppose $\alpha(f)>0$. If $\delta(f)=0$, then $f$ has a positive critical point less than $x_{1}(f)$ and the first inequality follows from Rolle's Theorem. Finally by Descartes' Rule of Signs, $\beta(f)=\alpha\left(f^{\prime}\right) \leq \gamma\left(f^{\prime}\right)=\gamma(f)-\delta(f)$.

Lemma B. If $\alpha(f)=\gamma(f)$, then $\alpha\left(f^{\prime}\right)=\gamma\left(f^{\prime}\right)$.

Proof. By Lemma A, $\alpha\left(f^{\prime}\right)=\beta(f) \leq \gamma\left(f^{\prime}\right)$ with equality if $\alpha(f)=\gamma(f)$.

Proof of Lemma 2.2. Let $w=\gamma(g)$. Assume $\alpha(h)>0$, otherwise Lemma 2.2 is trivial. Thus $\gamma(h)>0$, so $\gamma(f)=\alpha(f) \geq w+2$. Therefore $x_{w+2}(f)$ and $x_{1}(h)$ exist. Since $h(0)=0, \alpha\left(h^{\prime}\right)>0$ so $x_{1}\left(h^{\prime}\right)$ also exists. Note that $h(x)>0$ for $0<x<x_{1}(h)$. It suffices to prove

$$
x_{w+2}(f)<x_{1}(h) \text { and } g(x)<0 \text { for } x>x_{w+1}(f),
$$

for (10) implies that $g(x)<0$ for $x>x_{1}(h)$, whence $h>0$ at each zero of $f$ exceeding $x_{1}(h)$.

We can assume $g(0)$ is nonzero, otherwise divide $f$ by an appropriate power of $x$. Assume $g$ is nonconstant, otherwise $g$ is a negative constant, $w=0$, and (10) follows easily from Lemma A. Thus $g(x)=a+b x^{m}+\cdots$ where $m>0, a b$ is nonzero, and the three dots represent terms of degree greater than $m$. Since $g(x)$ has a negative leading term, $w$ is even or odd according as $a<0$ or $a>0$.

Note also that $\gamma\left(g^{\prime}\right)=w$ or $w-1$ according as $a b>0$ or $a b<0$.

Clearly (10) holds if $\operatorname{deg}(f)=3$, so assume $\operatorname{deg}(f)>3$. By Lemma B and induction on $\operatorname{deg}(f)$, we may suppose that

$$
\begin{gathered}
x_{w+2}\left(f^{\prime}\right)<x_{1}\left(h^{\prime}\right) \text { and } g^{\prime}(x)<0 \text { for } x>x_{w+1}\left(f^{\prime}\right), \quad \text { if } a b>0 ; \\
x_{w+1}\left(f^{\prime}\right)<x_{1}\left(h^{\prime}\right) \text { and } g^{\prime}(x)<0 \text { for } x>x_{w}\left(f^{\prime}\right), \quad \text { if } a b<0 .
\end{gathered}
$$

First suppose $a b>0$. If $a, b>0$, then $f, f^{\prime}>0$ for small $x>0$, so $f>0$ for $x_{i+1}(f)<x<x_{i+2}(f)$ when $i$ is odd. If $a, b<0$, then $f, f^{\prime}<0$ for small $x>0$, so $f>0$ for $x_{i+1}(f)<x<x_{i+2}(f)$ when $i$ is even. In either case, by (11),

$$
f(x)>0 \text { for } x_{w+1}(f)<x<x_{w+2}(f) .
$$

By Lemma A,

$$
x_{1}\left(f^{\prime}\right) \leq x_{1}(f) \leq \cdots \leq x_{w+1}\left(f^{\prime}\right)<x_{w+1}(f) \leq x_{w+2}\left(f^{\prime}\right) \leq x_{w+2}(f) .
$$

Thus by (12), $g$ is decreasing for $x>x_{w+1}(f)$. By (15) and (12), $x_{w+1}(f)<$ $x_{1}\left(h^{\prime}\right)<x_{1}(h)$, so $g\left(x_{w+1}(f)\right)=-h\left(x_{w+1}(f)\right)<0$. Thus for $x>x_{w+1}(f)$, we have $g<0$, i.e., $h>f$. It follows from (14) that $x_{w+2}(f)<x_{1}(h)$. This completes the proof of (10) when $a b>0$.

Finally, suppose $a b<0$. If $a<0$, then $f<0, f^{\prime}>0$ for small $x>0$, while if $a>0$, then $f>0, f^{\prime}<0$ for small $x>0$. In either case, we again see from (11) that (14) holds. By Lemma A,

$$
x_{1}(f)<x_{1}\left(f^{\prime}\right) \leq \cdots \leq x_{w}\left(f^{\prime}\right) \leq x_{w+1}(f)<x_{w+1}\left(f^{\prime}\right) \leq x_{w+2}(f) .
$$


Thus by (13), $g$ is decreasing for $x>x_{w+1}(f)$. By (16) and (13), $x_{w+1}(f)<$ $x_{1}\left(h^{\prime}\right)<x_{1}(h)$, so $g\left(x_{w+1}(f)\right)=-h\left(x_{w+1}(f)\right)<0$. Thus for $x>x_{w+1}(f)$, we have $g<0$, i.e., $h>f$. It follows from (14) that $x_{w+2}(f)<x_{1}(h)$. This completes the proof of (10) and Lemma 2.2.

\section{ACKNOWLEDGMENTS}

We wish to thank the mathematics department at the University of California at San Diego and the Mathematical Sciences Research Institute at Berkeley for supporting the first and last authors respectively as visiting scholars during some of the preparation time of this paper. We especially wish to thank the number theorist R. Evans of the University of California at San Diego for the considerably shortened version of the proof for Lemma 2.2 and the referee for many helpful suggestions.

\section{REFERENCES}

1. B. Beauzamy, Jensen's inequality for polynomials with concentration at low degrees, Numer. Math. 49 (1986), 221-225.

2. B. Beauzamy and P. Enflo, Estimations de produits de polynômes, J. Number Theory 21 (1985), 390-412.

3. R. Evans and J. Greene, Polynomials with positive coefficients whose zeros have modulus one, SIAM J. Math. Anal. 22 (1991).

4. R. Evans and P. Montgomery, Problem 6631, Amer. Math. Monthly 97 (1990), 432-433.

5. G. Polya and G. Szego, Problems and theorems in analysis II, Springer-Verlag, 1976.

6. A. K. Rigler, S. Y. Trimble, and R. S. Varga, Sharp lower bounds for a generalized Jensen inequality, Rocky Mountain J. Math. (1991).

Department of Mathematics, Texas Tech University, Lubbock, TeXas 79409

Current address, W. Dayawansa: Department of Electrical Engineering, University of Maryland, College Park, Maryland 20742 